\title{
Mevalonate pathway as a novel target for the treatment of metastatic gastric cancer
}

\author{
NATALIA ORTIZ ${ }^{1}$ and CECILIA DÍAZ ${ }^{1,2}$ \\ ${ }^{1}$ Department of Biochemistry, School of Medicine; ${ }^{2}$ Institute Clodomiro Picado, Faculty of Microbiology, \\ University of Costa Rica, San Pedro de Montes de Oca, San José 11501-2060, Costa Rica
}

Received March 17, 2020; Accepted September 1, 2020

DOI: $10.3892 / \mathrm{ol} .2020 .12183$

\begin{abstract}
Gastric mucosa tumors may present as two distinct major entities: Diffuse and intestinal subtypes. There is no standard treatment for advanced or metastatic gastric cancer. The mevalonate pathway and cholesterol homeostasis are important processes in cancer cells that may be highly relevant in terms of cell growth, survival and metastatic potential. Two model cell lines representing intestinal (NCI-N87) and diffuse (Hs746T) metastatic gastric tumor histological subtypes were treated with different drugs that alter membrane lipid metabolism to determine whether cell proliferation, viability and migration were affected. The results indicated that the cells exhibited significant differences in proliferation when treated with the cholesterol-lowering drug simvastatin, but not with terbinafine, another compound that affects cholesterol synthesis. Only simvastatin affected migration in both cell lines. Reposition studies with mevalonolactone, farnesyl pyrophosphate and geranylgeranyl pyrophosphate in the presence of high and low FBS concentrations indicated that both isoprenoids and cholesterol reversed the antiproliferative effects of simvastatin in gastric cancer cells. The cell lines used in the present study had different sensitivities to several potential anti-neoplastic agents that affect the synthesis of membrane lipids. The diffuse gastric cancer cells were particularly sensitive to simvastatin, suggesting it as an option for combination treatment.
\end{abstract}

\section{Introduction}

Gastric cancer is the fifth most common tumor type worldwide, with more than one million newly diagnosed cases reported each year and considered to be the third-leading cause of death by cancer (1). Although several classifications have been

Correspondence to: Professor Cecilia Díaz, Institute Clodomiro Picado, Faculty of Microbiology, University of Costa Rica, San Pedro de Montes de Oca, San José 11501-2060, Costa Rica

E-mail: cecilia.diaz@ucr.ac.cr

Key words: metastasis, gastric cancer, statins, isoprenoids, cholesterol suggested for gastric adenocarcinoma, the most widely used classification is based on Lauren's histological criteria, which divide gastric mucosa tumors into two distinct main entities: Diffuse and intestinal subtypes $(2,3)$.

The standard treatment for primary gastric cancer is radical surgery. However, as that the incidence of hematogenous and peritoneal metastasis is high, systemic chemotherapy is also part of the protocol, which does not discriminate between the two types of gastric carcinoma, mainly resulting in poor survival outcomes (4-7).

In addition, there is no standard treatment for advanced or metastatic gastric cancer. Chemotherapeutic treatment consisting of several drug combinations, including 5-fluorouracil or capecitabine together with oxaliplatin or cisplatin, are currently being used. These treatments are occasionally used in combination with either an anthracycline or docetaxel and irinotecan $(3,8,9)$. Although several meta-analyses have indicated that drug combinations result in better outcomes compared to single-agent chemotherapy (1 month above the 6.7 months of additional overall survival observed with monotherapy), clinically effective doses result in high toxicity for the patient $(9,10)$. Targeted therapy in combination with chemotherapy, mainly for HER2-positive disease (10-30\% of patients with gastric cancer), is also the first-line treatment for this specific group, but patients risk developing rapid tumor resistance to monoclonal antibody-based therapy (10-14).

Several studies suggested that cancer cells have higher requirements for cholesterol (15-17) and have elevated levels of membrane cholesterol rich-lipid rafts compared to normal cells. In addition, it was hypothesized that altering these microdomains, such as cyclodextrin-induced cholesterol depletion, may be a potential approach to treat cancer metastasis (18).

Membrane cholesterol is produced in the mevalonate pathway, which is one of the most important biosynthetic processes in animal cells (19-21). This sterol and other lipids derived from this pathway, such as isoprenoid groups that post-translationally modify membrane proteins, have been implicated in the survival and metastatic behavior of several types of cancer (22). Inhibitors of this pathway, such as plasma cholesterol-lowering statins, which inhibit the first rate-limiting enzyme hydroxy methyl glutaryl CoA reductase (HMGCR) and exert in vitro anti-proliferative and pro-apoptotic effects (21), have been clinically tested in patients with cancer $(20,22,23)$. Other drugs that interfere with the mevalonate pathway, such 
as zoledronic acid and farnesyl and geranylgeranyl transferase inhibitors that affect protein isoprenylation, have also been tested. Terbinafine, an inhibitor of the mevalonate pathway squalene epoxidase (24), was suggested to be a possible treatment option for several hepatocellular carcinoma tumors (25).

The present study assessed the toxic activity and growth and migration inhibition of agents that affect membrane lipid synthesis in cell lines widely used as models for advanced-stage intestinal and diffuse gastric carcinomas, which represent two genetic and phenotypically-different molecular tumors. The present study indicated their differential sensitivity to several potentially effective anticancer agents.

\section{Materials and methods}

Cell culture. NCI-N87 (ATCC CRL-5822) and Hs746T (ATCC HTB-135) cell lines were obtained from the American Type Culture Collection. The two cell lines were established from gastric carcinomas that metastasized to the liver (NCI-N87) and the left leg muscle (Hs746T). The NCI-N87 cell line is derived from an intestinal gastric tumor, whereas Hs746T cells originate from a diffuse gastric tumor. The cells were maintained in RPMI-1640 medium (cat. no. R8758; Sigma-Aldrich; Merck KGaA) supplemented with 10\% FBS (cat. no. F2442; Sigma-Aldrich; Merck KGaA) and 100 U/ml penicillin-streptomycin (cat. no. 15140122; Gibco; Thermo Fisher Scientific, Inc.) at $37^{\circ} \mathrm{C}$ in a humidified atmosphere containing $5 \% \mathrm{CO}_{2}$.

Growth curve analysis. Initially, $1.5 \times 10^{4}$ cells were seeded in 24-wells plates. Cells were counted using a hemocytometer in a 1:2 dilution with Trypan blue every 2 days. For each cell line, the dividing time (DT) between days 2 and 4 was determined using the following formula: $\mathrm{DT}=\mathrm{T} \ln 2 / \ln (\mathrm{Xf} / \mathrm{Xi})$, where $\mathrm{T}$ is the incubation time, $\mathrm{Xf}$ the final cell number and $\mathrm{Xi}$ the initial number of cells (26).

Cell viability assay. An MTT assay was used to determine the effect of various drugs on the metastatic gastric cancer cell lines. Once the cells were subjected to different treatments, the medium was removed and a solution of MTT in RPMI-1640 medium $(0.5 \mathrm{mg} / \mathrm{ml})$ was added. Cells were incubated for $2 \mathrm{~h}$ and the medium was subsequently removed. Precipitated formazan crystals were dissolved in $95 \%$ ethanol. Cells that were incubated with medium alone were used as a control and defined as having $100 \%$ viability. Absorbance values were determined at a wavelength of $570 \mathrm{~nm}$ using a microplate reader (BioTek Cytation 3 Imaging Multi-Mode Reader; BioTek Instruments, Inc.).

Cisplatin-induced cytotoxicity. NCI-N87 (1.6x10 4$)$ and Hs746T $\left(8 \times 10^{3}\right)$ cell suspensions were cultured in 96-well plates and allowed to attach overnight. Cells were incubated with different concentrations of cisplatin solution $(6-500 \mu \mathrm{M}$; Pfizer, Inc.) in 10\% FBS supplemented with RPMI-1640 medium for $48 \mathrm{~h}$. The viability assay was then performed as mentioned above.

Inhibition of HMGCR. Simvastatin (cat. no. S6196; Sigma-Aldrich; Merck KGaA) was used to inhibit HMGCR. To activate the drug, the protocol described by Dong et al (27)
Table I. $\mathrm{IC}_{50}$ values for cisplatin and simvastatin on Hs746T and NCI-N87 cells for $48 \mathrm{~h}$.

\begin{tabular}{lrr}
\hline Drug & Hs746T & NCI-N87 \\
\hline Cisplatin & $39.2 \pm 1.8$ & $26.8 \pm 2.3^{\mathrm{a}}$ \\
Simvastatin & $2.3 \pm 0.2$ & $141.7 \pm 2.8^{\mathrm{b}}$ \\
\hline
\end{tabular}

${ }^{\mathrm{a}} \mathrm{P}<0.05$ and ${ }^{\mathrm{b}} \mathrm{P}<0.001$; comparison of $\mathrm{IC}_{50}$ values for each treatment between cell lines. Values are expressed as the mean \pm standard error of three independent experiments performed in triplicate.

was used. NCI-N87 $\left(1.6 \times 10^{4}\right)$ and Hs746T $\left(8 \times 10^{3}\right)$ cell suspensions were cultured in 96-well plates and allowed to attach overnight. Cells were incubated with different concentrations of the drug (3-100 $\mu \mathrm{M})$ for $48 \mathrm{~h}$ in $10 \%$ FBS supplemented RPMI-1640 medium. This range of simvastatin concentrations was based on previous studies $(28,29)$.

Mevalonolactone (1.25 $\mu \mathrm{M}$; cat. no. M4667; Sigma-Aldrich; Merck KGaA) and the isoprenoids geranylgeranyl pyrophosphate (GGPP; cat. no. G6025; Sigma-Aldrich; Merck KGaA) and farnesyl pyrophosphate (FPP; cat. no. F6892; Sigma-Aldrich; Merck KGaA) were used to evaluate the effect of intermediary metabolites of the mevalonate pathway. Cells were incubated simultaneously for $48 \mathrm{~h}$ with simvastatin and metabolites in medium supplemented with $10 \%$ FBS. In addition, the effect of the incorporation of the metabolites in low-cholesterol media was evaluated using Advanced RPMI media (cat. no. 12633012; Thermo Fisher Scientific, Inc.) containing $1 \%$ FBS.

Inhibition of squalene epoxidase. Terbinafine (cat. no. T8826; Sigma-Aldrich; Merck KGaA) was dissolved in DMSO to a final concentration of $150 \mathrm{mM}$. The same number of cells used for simvastatin treatment was incubated for $48 \mathrm{~h}$ with different concentrations of inhibitor $(5-150 \mu \mathrm{M})$ dissolved in medium supplemented with $10 \%$ FBS. The DMSO concentration in the medium was $<0.1 \%$, which was not toxic to the cells. For the control groups, cells were incubated with the same DMSO concentrations utilized in the treatment groups. This range of terbinafine concentrations was based on previous studies $(30,31)$. Cell viability at $48 \mathrm{~h}$ in the presence of terbinafine in Advanced RPMI media containing 1\% FBS was also assessed.

Membrane cholesterol staining. NCI-N87 and Hs746T cells were plated in 96-black-well plates. Subsequently, cells were treated with inhibitors, simvastatin or terbinafine for $48 \mathrm{~h}$ at the $\mathrm{IC}_{50}$ value. Cells were fixed with $4 \%$ paraformaldehyde for $10 \mathrm{~min}$ at room temperature. Cells were then incubated in $1.5 \mathrm{mg} / \mathrm{ml}$ glycine solution for $10 \mathrm{~min}$ at room temperature. Subsequently, the cells were incubated in $0.05 \mathrm{mg} / \mathrm{ml}$ solution of filipin (cat. no. F9765; Sigma-Aldrich; Merck KGaA) in PBS supplemented with $10 \%$ FBS for $2 \mathrm{~h}$ at room temperature and protected from light. As a positive control for the staining, cells were incubated with $5 \mathrm{mM}$ methyl- $\beta$-cyclodextrin (MßCD; cat. no. C4555; Sigma-Aldrich; Merck KGaA) for $1 \mathrm{~h}$ to extract cholesterol from the plasma membrane. Images 
A

Hs746T: Diffuse tumor

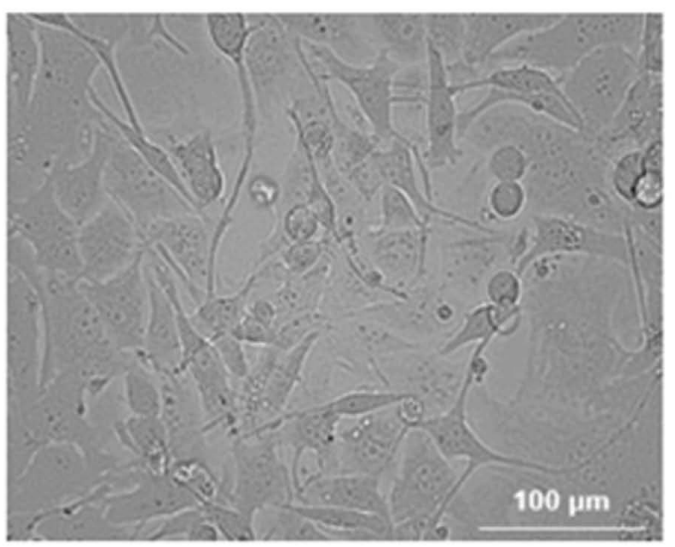

Leg (muscle) metastasis

Differentiation: high

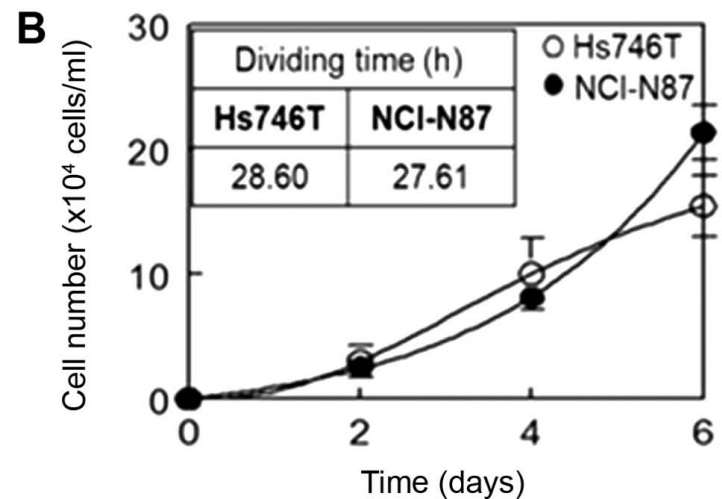

NCI-N87: Intestinal tumor

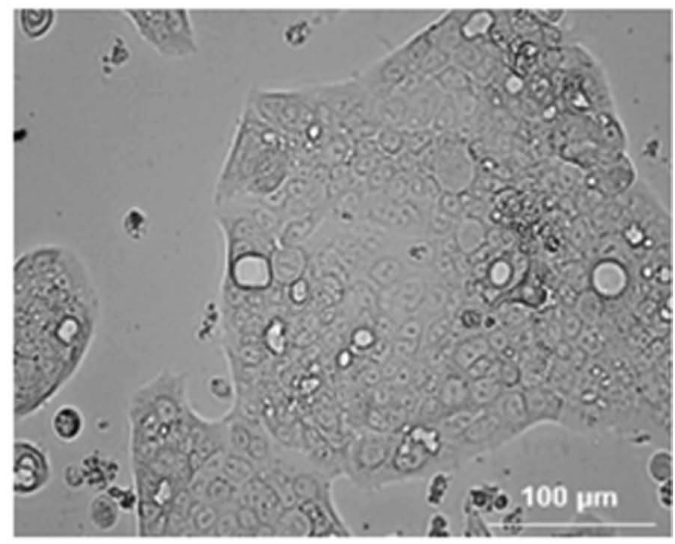

Liver metastasis

Differentiation: high

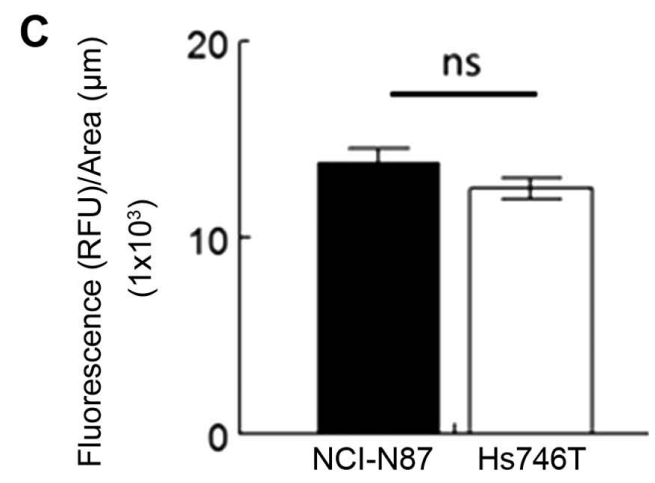

Figure 1. Major characteristics of the two metastatic gastric cancer cell lines. (A) Microscopy images displaying the morphological features (scale bar, $100 \mu$ m). Hs746T cells derive from a diffuse tumor and were obtained from a metastatic site (left leg). NCI-N87 cells have conserved properties of an intestinal tumor and are derived from a gastric metastasis to the liver. (B) Growth curve and cell division rates of the two metastatic cell lines. (C) Membrane cholesterol content in NCI-N87 and Hs746T cells measured by filipin staining. Ns, no significance.

were acquired by fluorescence microscopy with a x20 objective under the same conditions of LED exposure and gain on a BioTek Cytation 3 Imaging Multi-Mode Reader.

Gen5 Image+ 3.09 software (BioTek Instruments, Inc.) was used to quantify the fluorescence intensity. An experiment for each cell line that included all the images (control and treatments) was set and automatic image preprocessing for DAPI and Brightfield channels were applied. The Cellular Analysis Tool was used to select the areas that contained only cells in the Brightfield images. For experiments with Hs746T cells, the threshold value was set to 7,000 and object size selection was between 20 and $200 \mu \mathrm{m}$. For NCI-N87 cells, the parameters were set to a threshold value of 4,000 and object size selection was between 20 and $500 \mu \mathrm{m}$. For both cell lines, the primary edge objects were included, and the entire image was analyzed.

The Object Sum Intensity [Tsf (DAPI 377,447)] and the Object Sum Area [Tsf (Bright Field)] were estimated using the software. For each experiment, 4-11 different images were analyzed. The relative Object Sum Intensity/Object Sum Area was estimated for all images. The mean value was determined, and statistical analyses were performed.
Wound-healing assay and inhibition of cell migration. NCI-N87 and Hs746T cells were grown on 24-well plates at a density of $1 \times 10^{6}$ and $3 \times 10^{5}$ cells/well, respectively, and allowed to adhere overnight in the presence of $10 \%$ FBS. Wounds were scraped in the middle of the well with a sterile pipette tip. Prior to treatment, cell monolayers were washed twice with culture media to remove floating cells. Treatment with simvastatin $(10 \mu \mathrm{M})$ and terbinafine $(20 \mu \mathrm{M})$ was performed in duplicate samples for each cell line in media containing 10\% FBS. A total of 2 light microscopy images were captured on the BioTek Cytation 3 from two visual fields of the wells. Images were acquired at 0 and $24 \mathrm{~h}$ for Hs746T cells and 0 and $48 \mathrm{~h}$ for NCI-N87 cells. The percentage of migration was calculated by the average obtained from three random measurements and expressed as a percentage of the control (time 0 for each group) using ImageJ v1.49 software (National Institutes of Health).

Sulforhodamine B assay. To determine the effect on cell proliferation of simvastatin and terbinafine during the aforementioned wound-healing assay, as well as the effect of the presence of FBS in the media, the Sulforhodamine B assay 

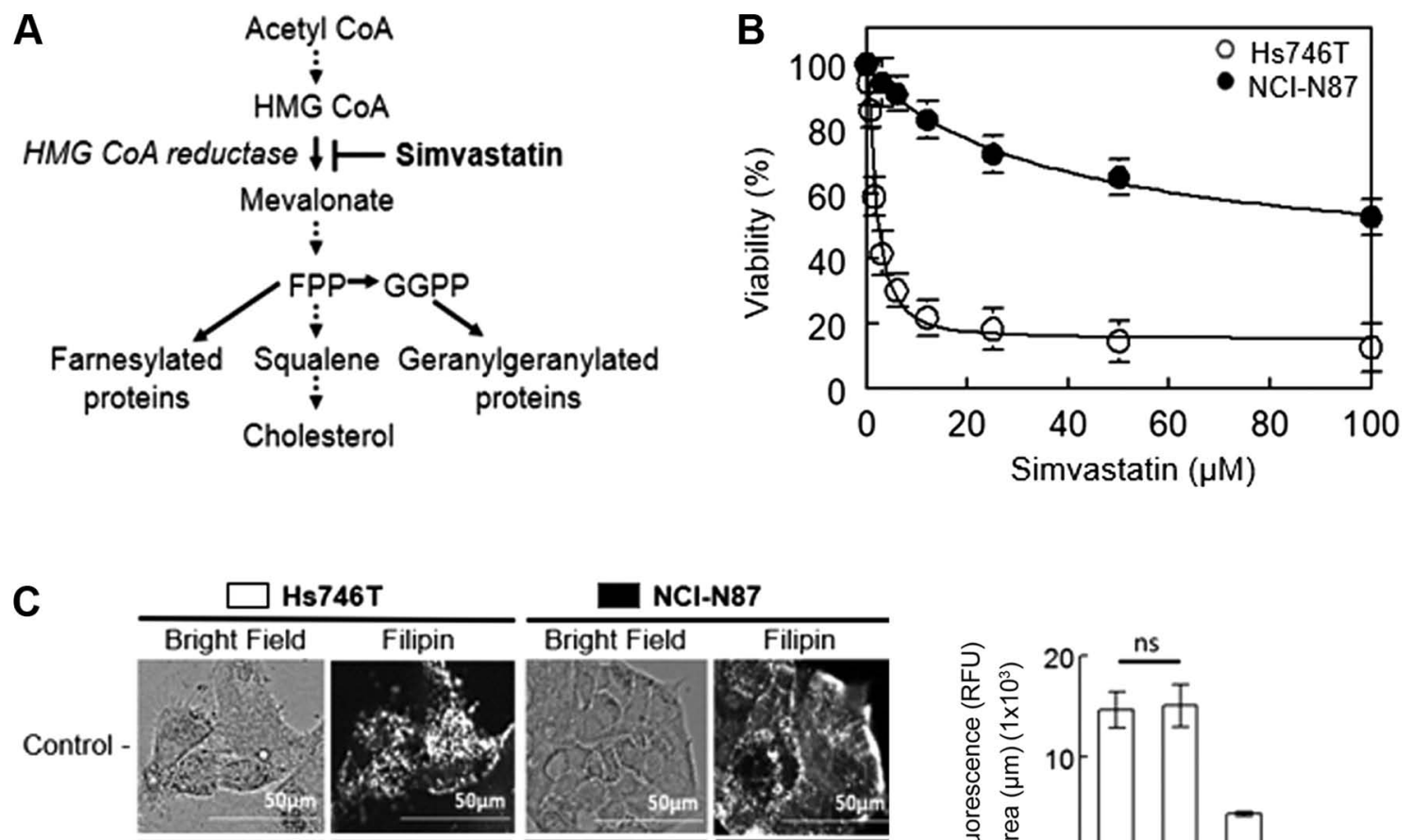

Hs746T
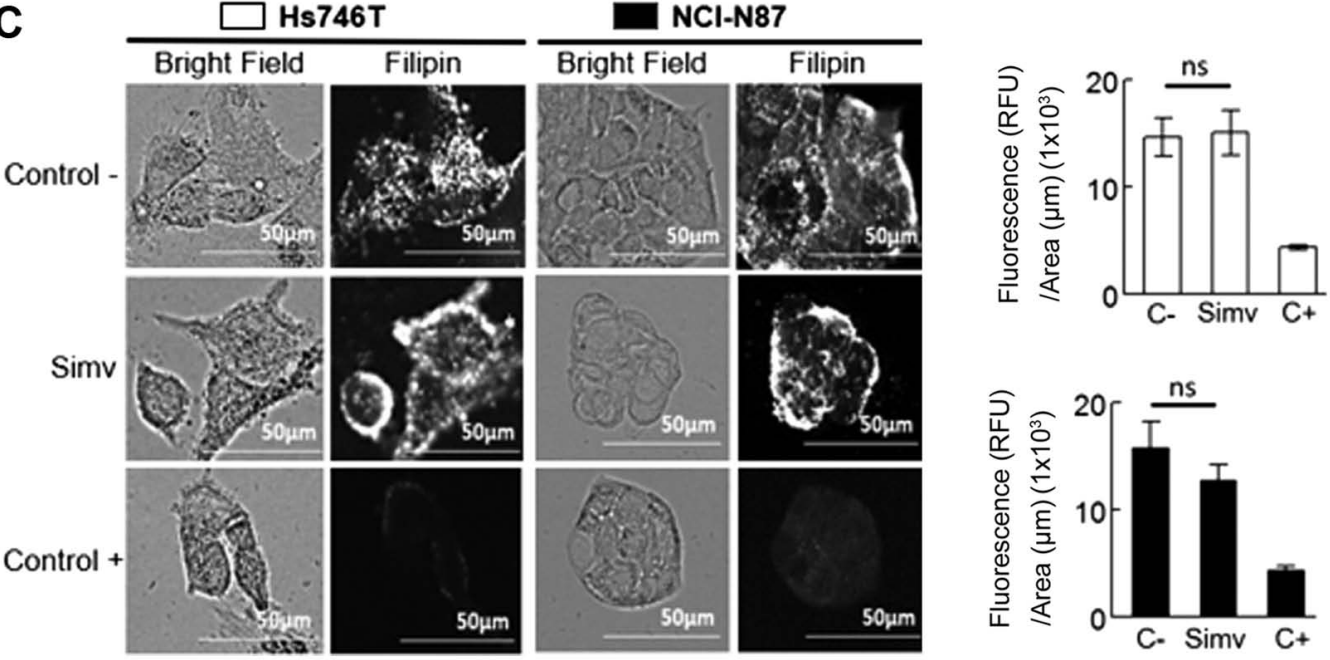

Figure 2. Inhibitory effect of HMGCR on Hs746T and NCI-N87 cell viability. (A) Schematic explaining the target site of simvastatin on the mevalonate pathway. (B) Dose-response curve of both cell lines treated with simvastatin for $48 \mathrm{~h}$. Values are expressed as the mean \pm standard error of three independent experiments performed in triplicate. (C) Left panel: Visualization of membrane cholesterol by filipin staining after both cell lines were incubated with simvastatin at the $\mathrm{IC}_{50}$ value for $48 \mathrm{~h}$. As a positive control, cells were treated with $5 \mathrm{mM} \mathrm{M \beta CD}$ to determine a decrease in cell membrane cholesterol (scale bars, $50 \mu \mathrm{m}$ ). Right panel: Fluorescence quantification adjusted by area. Values are expressed as the mean \pm standard error of $\geq 4$ independent wells. HMGCR, hydroxymethyl glutaryl CoA reductase; FPP, farnesyl pyrophosphate; GGPP, geranylgeranyl pyrophosphate; M $\beta C D$, methyl- $\beta$-cyclodextrin; simv, simvastatin; $\mathrm{C}-$, negative control; $\mathrm{C}+$, positive control; Ns, no significance.

was performed as previously described (32). Treated cells were fixed in $10 \%$ trichloroacetic acid for $1 \mathrm{~h}$ at $4^{\circ} \mathrm{C}$ and stained with $0.04 \%$ Sulforhodamine B solution (cat. no. S1402; Sigma-Aldrich; Merck KGaA) for $30 \mathrm{~min}$ at room temperature. Subsequently, the wells were rinsed with $1 \%$ acetic acid to remove the excess dye. Protein-bound dye was dissolved in $10 \mathrm{mM}$ Tris base solution and the absorbance was measured at $530 \mathrm{~nm}$, using a microplate reader (BioTek Cytation 3 Imaging Multi-Mode Reader). To determine the percentage of cell proliferation relative to a non-treated control, cells without treatment were also analyzed.

Statistical analysis. For all cell viability assays, dose-response curves were plotted and the $\mathrm{IC}_{50}$ value was calculated using Slide Write Plus 6.10 software (Advanced Graphics Software, Inc). To evaluate the significance of differences in results between two groups, an independent t-test was performed. One-way ANOVA was performed to compare three or more groups, followed by Bonferroni's post-hoc test. All results were analyzed with GraphPad Prism 5 (GraphPad Software, Inc.) and SPSS 22 software (IBM Corp.).

\section{Results}

Characteristics of metastatic gastric cancer cell lines. Two model cell lines representing the two major subtypes of gastric metastatic carcinoma were characterized in terms of proliferation and cholesterol content (Fig. 1). Both cell lines, derived from different metastasis sites, exhibited significant differences in morphology and levels of differentiation (Fig. 1A). The two cell lines had a similar proliferation rate (Fig. 1B) and membrane basal cholesterol levels (Fig. 1C).

Cisplatin and mevalonate pathway inhibitors induce cytotoxicity. The effect of the chemotherapeutic drug cisplatin (commonly used in the treatment of gastric cancer) on the viability of Hs746T and NCI-N87 cells was included for comparison. In terms of $\mathrm{IC}_{50}$ values at $48 \mathrm{~h}$, there was a significant difference between the two cell lines (Table I), with NCI-N87 having a greater sensitivity to the drug.

Upon inhibition of HMGCR with simvastatin (Fig. 2A), there was a strong effect on Hs746T cells, whereas NCI-N87 cells were highly resistant. At $48 \mathrm{~h}$ of treatment with the drug, 
A
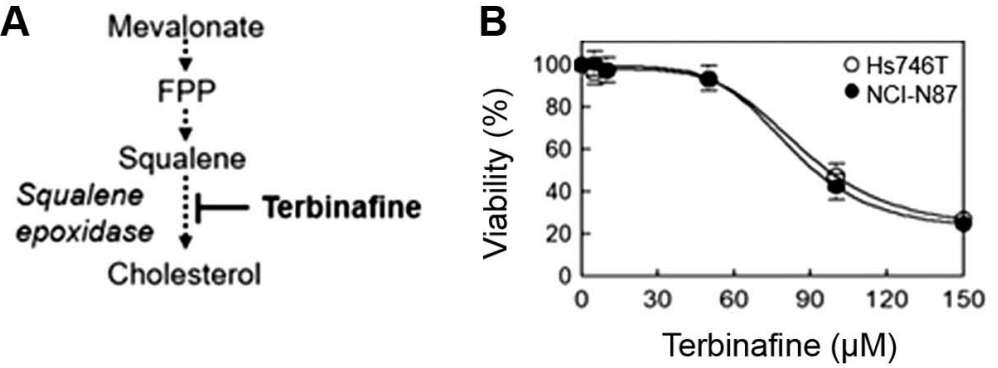

D
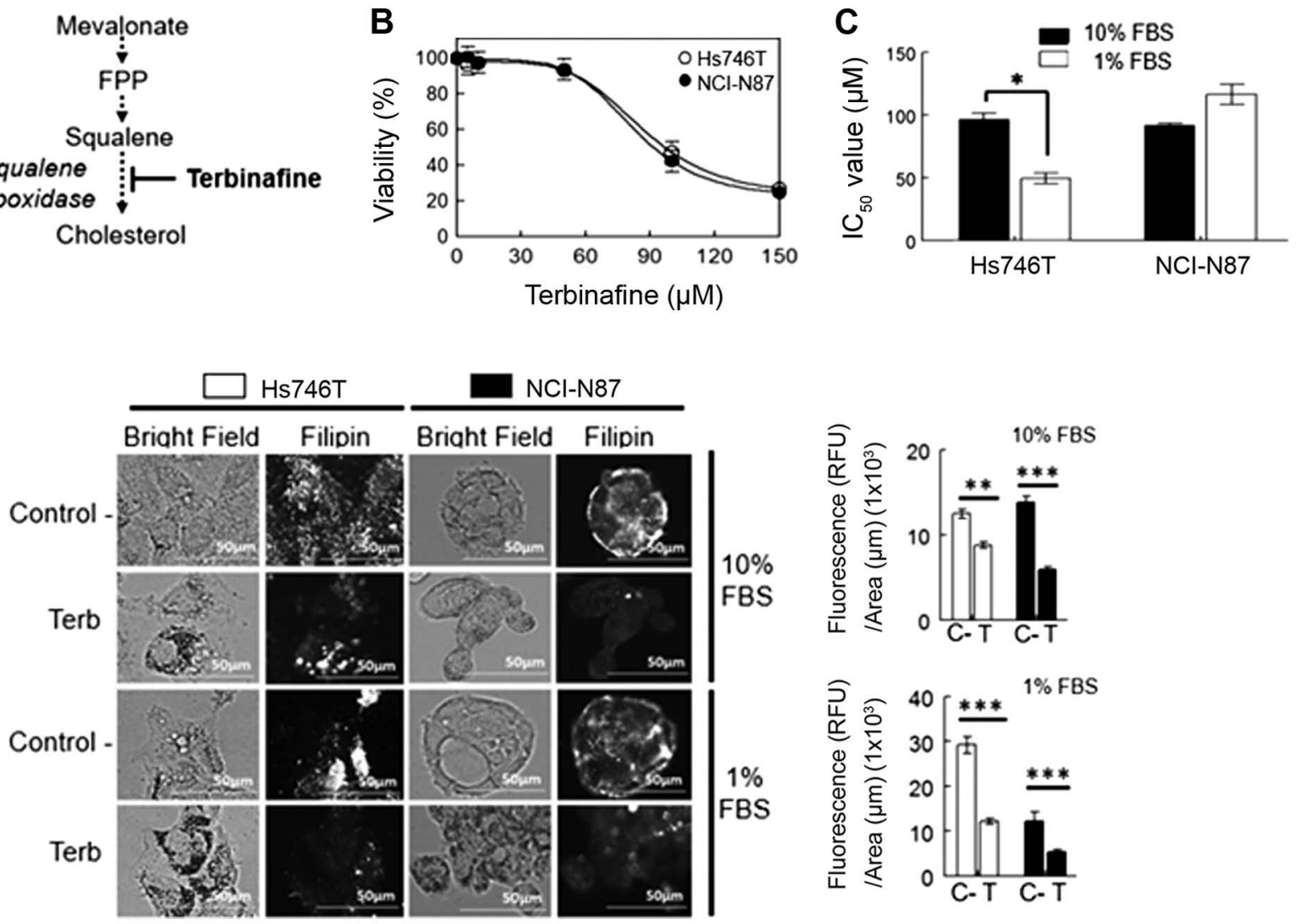

Figure 3. Effects of squalene epoxidase inhibition on Hs746T and NCI-N87 cell viability. (A) Schematic explaining the target site of terbinafine on the

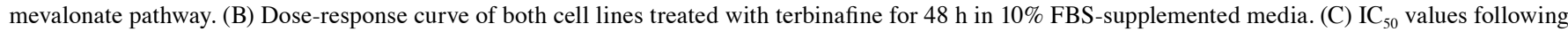
terbinafine treatment for $48 \mathrm{~h}$ in media supplemented with 1 or $10 \%$ FBS. Values are expressed as the mean \pm standard error of three independent experiments performed in triplicate. (D) Left panel: Visualization of membrane cholesterol by filipin staining after both cell lines were incubated with terbinafine at the $\mathrm{IC}_{50}$ value for $48 \mathrm{~h}$ in media supplemented with 1 or $10 \%$ FBS (scale bars, $50 \mu \mathrm{m}$ ). Right panel: Fluorescence quantification adjusted by area. Values are expressed as the mean \pm standard error of $\geq 4$ independent wells. ${ }^{*} \mathrm{P}<0.05,{ }^{* *} \mathrm{P}<0.01$ and ${ }^{* * *} \mathrm{P}<0.001$. FPP, farnesyl pyrophosphate; T/Terb, terbinafine; $\mathrm{C}$, control.

the $\mathrm{IC}_{50}$ on Hs746T cells was $2.3 \pm 0.2 \mu \mathrm{M}$, whereas the $\mathrm{IC}_{50}$ on NCI-N87 cells was $141.7 \pm 2.8 \mu \mathrm{M}$ (Table I; Fig. 2B). Despite the evident difference in cell viability, when staining the cells with the macrolide filipin (which binds to free cholesterol) to demonstrate the effect of simvastatin on cholesterol metabolism, the staining intensity was statistically similar to that of the negative controls in both cell lines (Fig. 2C). The negative controls corresponded to cells without simvastatin treatment, and treatment with methyl- $\beta$-cyclodextrin, a cholesterol chelator, was used as a positive control. Of note, all experiments were performed in media supplemented with $10 \%$ FBS, which allowed the cells to uptake cholesterol from the media.

Upon treatment with terbinafine, which is an inhibitor of squalene epoxidase (Fig. 3A), no significant differences were observed in the $\mathrm{IC}_{50}$ values between both cell lines in the media supplemented with $10 \%$ FBS (Fig. 3B). Reducing the concentration of FBS to $1 \%$ increased the toxicity of the drug in the diffuse gastric tumor Hs746T cell line (Fig. 3C). Upon staining of cholesterol with filipin following terbinafine treatment a significant reduction in cholesterol levels in both cell lines was observed, which was independent of the FBS concentration in the media (Fig. 3D).

Effects of treatment in the presence of intermediary metabolites mevalonolactone, FPP and GGPP. To determine the dependence of cholesterol and other mevalonate-derived molecules (isoprenoids) on cell viability, cells were co-incubated for $48 \mathrm{~h}$ with simvastatin in the presence of mevalonolactone, FPP or GGPP in media supplemented with 10 or $1 \%$ FBS.

The presence of mevalonolactone in the media resulted in the complete recovery of viability in both cell lines, independently of the FBS concentration. Furthermore, among the cells replenished with isoprenoids in media supplemented with $10 \% \mathrm{FBS}, 1.25 \mu \mathrm{M}$ GGPP was able to restore the viability only in Hs746T cells. For NCI-N87 cells, the dose was increased to $7.5 \mu \mathrm{M}$ to obtain partial recovery (data not shown), since $1.25 \mu \mathrm{M}$ did not result in any significant effects in media supplemented with $10 \%$ FBS. The addition of FPP to the media did not affect cell growth in the presence of $10 \% \mathrm{FBS}$, whereas addition of FPP or GGPP in the media containing 1\% FBS, resulted in the complete recovery of viability of Hs746T and NCI-N87 cells (Fig. 4).

Effects of simvastatin and terbinafine on cell migration. As presented in Fig. 5, simvastatin, but not terbinafine, was able to significantly inhibit the migration of both cell lines in media containing $10 \%$ FBS. For Hs746T cells, the incubation was performed for $24 \mathrm{~h}$ to avoid the higher toxicity of simvastatin at $48 \mathrm{~h}$. In addition, the cytotoxicity and cell proliferation were monitored through the entire wound-healing assay using the Sulforhodamine B assay (data not shown), and the control 

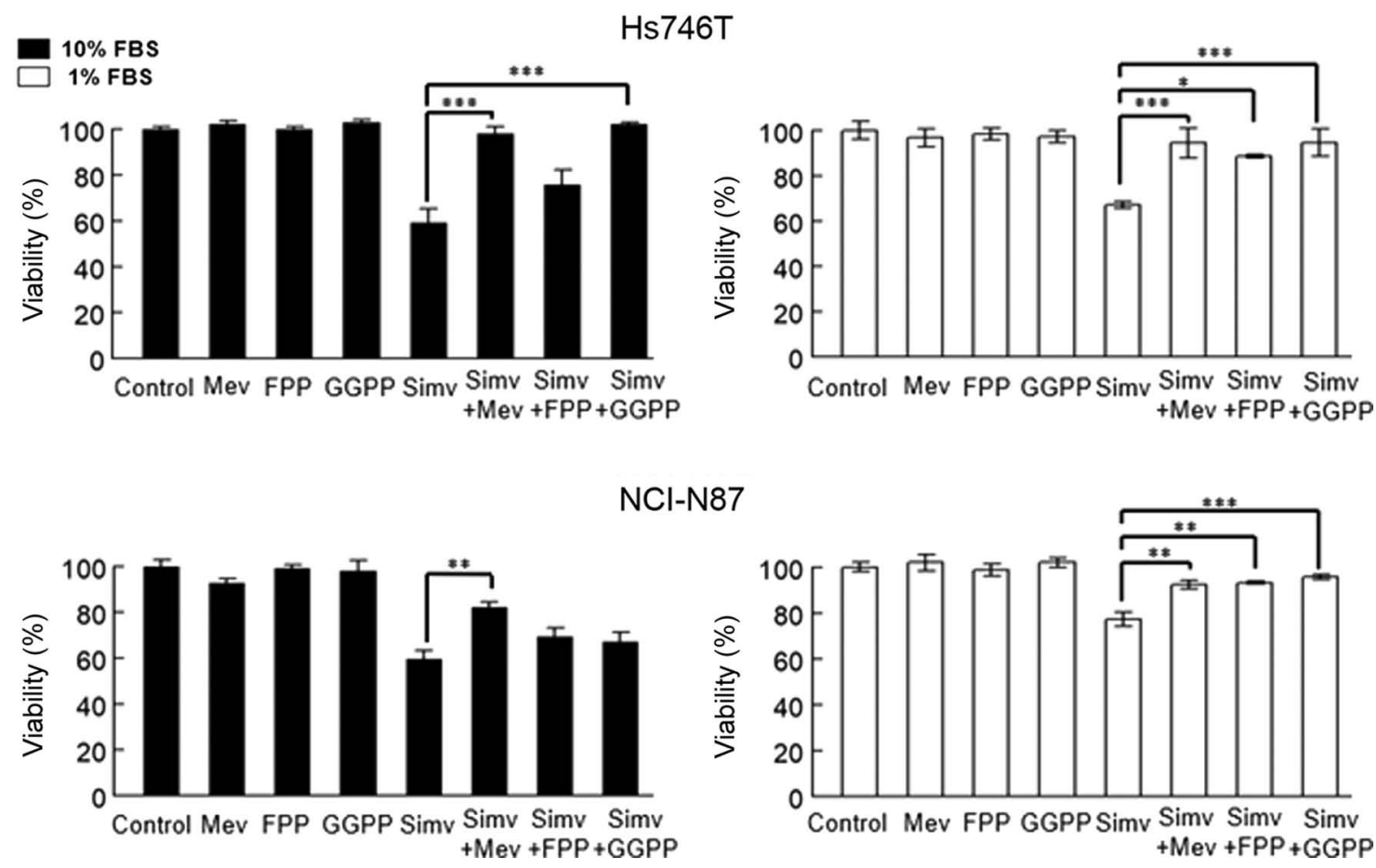

Figure 4. Replenishment of intermediary metabolites of the mevalonate pathway. Hs746T and NCI-N87 cells were co-incubated with simvastatin and the intermediaries mevalonolactone, FPP and GGPP for $48 \mathrm{~h}$ in media supplemented with 1 or $10 \%$ FBS. Values are expressed as the mean \pm standard error of three independent experiments performed in triplicate. ${ }^{*} \mathrm{P}<0.05,{ }^{* * *} \mathrm{P}<0.01$ and ${ }^{* * *} \mathrm{P}<0.001$. FPP, farnesyl pyrophosphate; GGPP, geranylgeranyl pyrophosphate; Simv, simvastatin; Mev, mevalonolactone.
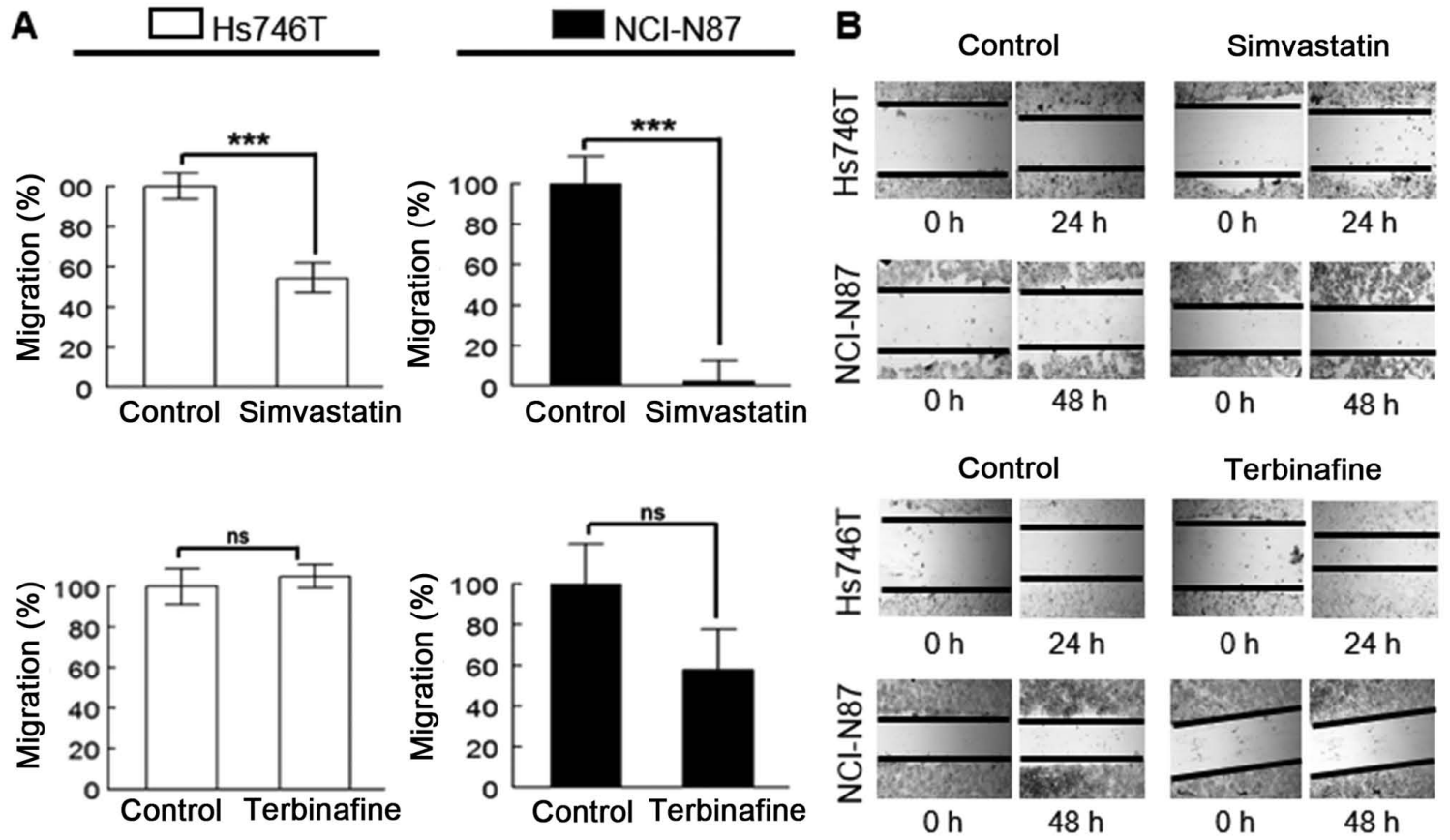

Terbinafine
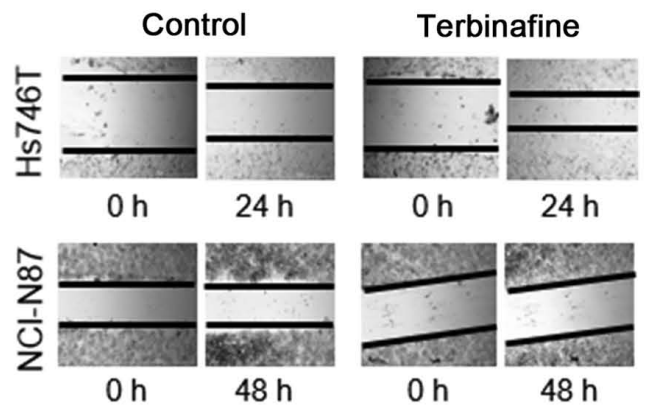

Figure 5. Migration of Hs746T and NCI-N87 cells treated with simvastatin and terbinafine. The effects of simvastatin $(10 \mu \mathrm{M})$ and terbinafine $(20 \mu \mathrm{M})$ on the migration of Hs746T (24 h) and NCI-N87 (48 h) were assessed using wound-healing assays. (A) The migration is expressed as the percentage of the wound width at the initial time-point (time 0 ) for both control and experimental treatments. Values are expressed as the mean \pm standard error of duplicate experiments. ${ }^{* * * *} \mathrm{P}<0.001$. (B) Representative images from the wound-healing assay of controls and cells treated with simvastatin or terbinafine for $24 \mathrm{~h}(\mathrm{Hs} 746 \mathrm{~T})$ and $48 \mathrm{~h}$ (NCI-N87).

groups were incubated with the same FBS concentration as the treatment groups. For NCI-N87 cells, the inhibition of migration was determined at $48 \mathrm{~h}$, as the cells migrated at a rate that was 4 times lower than that of Hs746T cells.

\section{Discussion}

Advanced intestinal and diffuse subtypes of gastric cancer are considered pathologically separate entities with different 
origins and causes. However, they are clinically treated similarly, even though patients should receive different therapies (3). One of the major differences between these two pathologies lies in their migration and invasion characteristics: The diffuse subtype is mainly associated with peritoneal dissemination, whereas the risk of liver metastasis is higher in the intestinal subtype, due to hematogenous dispersion (33).

Metastatic disease is practically incurable. In terms of chemotherapy options, diffuse tumors result in worse prognosis (34). Patients with the intestinal subtype that overexpress HER2 have the option of receiving monoclonal antibody therapy with trastuzumab (35). However, $\sim 50 \%$ of patients exhibit resistance to treatment (11).

In terms of cytotoxic agents used for chemotherapy of gastric cancer, the present study evidenced that clinically-used cisplatin alone exerted effects in both cell types, even when differences in the $\mathrm{IC}_{50}$ were observed. These results were expected, since platinum drugs are alkylating agents that bind to DNA and inhibit its replication. Although they target dividing cells at the beginning of DNA synthesis, they lack specificity, resulting in differences when cells are not synchronized $(36,37)$. Hs746T cells were more resistant to cisplatin treatment probably because, according to their proliferation rate, these cells did not exhibit exponential growth, which may be a limitation for the comparison. The lack of an exponential growth phase may be due to cell contact-inhibition mechanisms (38).

Highly proliferating cancer cells have higher requirements for cholesterol $(16,17)$. Statins have been used as chemopreventive agents and a treatment option for several tumor types (22). To satisfy their cholesterol requirements, cells may increase its endogenous synthesis or obtain cholesterol from outside through low density-lipoprotein (LDL) receptors (15). HMGCR, a mevalonate pathway first rate-limiting enzyme, has been considered as a metabolic oncogene. Although statins have been indicated to induce its transcriptional upregulation, several tumor types have deficient HMGCR feedback controls (39).

Caruso et al (40) reported that HMGCR activity is significantly higher in neoplastic tissues compared with that in normal gastric mucosa (in primary gastric tumors), whereas no differences were observed between diffuse and intestinal subtypes. They also suggested that LDL receptor levels were lower in primary gastric neoplastic tissues, but only in the diffuse type. This may explain the present results with simvastatin, since Hs746T diffuse tumor cells were more sensitive to the inhibitor compared to the intestinal type, NCI-N87 cells. However, the present study did not indicate a decrease in free cholesterol when both cell lines were treated with simvastatin, suggesting the triggering of a normal feedback that maintains cell cholesterol at normal levels.

In terms of treatment, it has been indicated that the statin concentration required to reach antitumoral therapeutic doses is 10 times higher than the doses utilized for the treatment of patients with hypercholesterolemia $(41,42)$. The doses of statins (such as simvastatin) used to treat hypercholesterolemia are in the range of 10-40 $\mathrm{mg}$ per day (43), or $0.2-0.6 \mathrm{mg} / \mathrm{kg} / \mathrm{day}$. Doses of $1 \mathrm{mg} / \mathrm{kg} /$ day would give a serum level of $\sim 0.1 \mu \mathrm{M}$, and therapeutic doses of $2-4 \mu \mathrm{M}$ are well tolerated in animal models (44). According to the present results, the $\mathrm{IC}_{50}$ of simvastatin on $\mathrm{Hs} 746 \mathrm{~T}$ cells was $2.3 \mu \mathrm{M}$, which is consistent with the aforementioned in vivo tolerated range, whereas the $\mathrm{IC}_{50}$ on NCI-N87 cells was much higher $(142 \mu \mathrm{M})$.

In the presence of $10 \% \mathrm{FBS}$, mevalonolactone and GGPP (but not FPP) led to recovery of the cells from simvastatin-induced toxicity. This indicates that isoprenoids are the most important factor for maintaining cell proliferation when HMGCR is inhibited under these conditions. However, when the FBS concentration was lowered to 1\%, FPP also led to the recovery from the cytotoxic effects, indicating that these cells require isoprenoids and cholesterol to proliferate normally. FPP is a downstream precursor metabolite for cholesterol and GGPP synthesis in the mevalonate pathway. Several proteins utilize FPP for farnesylation. However, to be converted into GGPP, FPP requires other metabolites that may not necessarily always be present in the cells (45). The differences in viability observed with FPP reposition at high and low levels of FBS suggest that FPP is being utilized to synthesize cholesterol.

Statins have been proposed as an option for the treatment of several tumor types $(20,22,23)$. Based on the present results, simvastatin may be an alternative treatment for the diffuse subtype of gastric carcinoma, even though certain effects may be due to other pleiotropic activities of the drug (46).

The present study revealed that the cells exhibited resistance upon treatment with the squalene-epoxidase inhibitor terbinafine, which affects the downstream second rate-limiting enzyme of the mevalonate pathway (24), in the presence of $10 \%$ FBS. However, Hs746T cells have a higher sensitivity in the presence of $1 \%$ FBS, indicating that cholesterol also influences cell viability. Conversely, this does not appear to be the case for NCI-N87 cells, which remained resistant to treatment.

The antifungal drug terbinafine has been proposed to be a potential agent for the treatment of several specific types of liver cancer (47). Terbinafine may also be an option for advanced gastric cancer treatment, although the high doses required may give rise to complications. In the present study, dark precipitates were observed in cells treated with terbinafine, similar to the squalene deposits observed in a previous study (47) and squalene-induced toxicity also occurred in these cancer cells.

Upon testing the effects of simvastatin and terbinafine on cell migration, it was indicated that the statin significantly affected both cell types. A comparison between cell lines was not possible due to the different incubation times used for each cell line, which was due to differences in drug sensitivity and basal migration ability of these cells. A limitation of the present study was that FBS was not removed from the media, and sera may induce cell proliferation, but due to the poor migratory ability observed (especially for NCI-N87), long time-points had to be used in these experiments, making it difficult to remove the sera due to loss of viability. However, controls were also in the presence of $10 \%$ FBS, which helped to correct for the potential effect induced by proliferation.

Terbinafine did not exert any significant effect on cell migration, suggesting that the effect of simvastatin may be due to the inhibition of protein prenylation. However, since the migration experiments were performed in the presence of $10 \% \mathrm{FBS}$, cholesterol may also have a role in the process. 
Prenylated proteins such as Rho are associated with migration and invasion of cancer cells (48). In addition, the role of HMGCR in gastric cancer cell migration has also been established (49).

Even when no normal gastric cells were used in the present study as a control, since they require specific nutrients and growth factors that may affect the interpretation of the data, the present study suggested that model cell lines representing the two advanced gastric tumor subtypes (intestinal and diffuse) respond differently to potential anti-neoplastic agents, including cholesterol and the isoprenoid-lowering drugs simvastatin and terbinafine. Preliminary results from our group on primary gastric tumor cells indicated a similar level of sensitivity to simvastatin as Hs746T cells (data not shown).

Another limitation of the present study is that only one cell line from each histological subtype of the tumor was tested; however, these are probably some of the best characterized gastric cancer cells commercially available and they have been widely used as a model of these subtypes of metastatic tumors $(50,51)$. They also have interesting features regarding the overexpression of two genes that are associated with tumor resistance, c-Met (Hs746T) and HER2 (NCI-N87). Even though the cell signaling pathways associated with these oncogenes were not characterized in the present study, this may be worth exploring in the future.

The present study provided possible novel therapeutic alternatives for advanced gastric cancer, alone or in combination with other drugs. The results also established that different drug treatment protocols should be provided for these two subtypes of gastric tumor, mainly in the advanced stages and in the presence of metastases. These two types of gastric tumors are different molecular entities at the genotypical and phenotypical level, and exhibit resistance to certain drugs and treatments. This may be due to their specific genetic alterations and levels of differentiation associated with epithelial-mesenchymal transition events. Hence, this should be taken into consideration during the selection of more appropriate and effective therapeutic options.

\section{Acknowledgements}

Not applicable.

\section{Funding}

This study was partially supported by Vicerrectoría de Investigación (grant no. 422-B7-098) and Sistema de Estudios de Posgrado, both from the Universidad de Costa Rica.

\section{Availability of data and materials}

All data generated or analyzed during this study were included in this published article.

\section{Authors' contributions}

NO and CD conceived and designed the study, analyzed the results and wrote and reviewed the manuscript. Both authors read and approved the manuscript.

\section{Ethics approval and consent to participate}

Not applicable.

\section{Patient consent for publication}

Not applicable.

\section{Competing interests}

The authors declare that they have no competing interests.

\section{References}

1. Bray F, Ferlay J, Soerjomataram I, Siegel RL, Torre LA and Jemal A: Global cancer statistics 2018: GLOBOCAN estimates of incidence and mortality worldwide for 36 cancers in 185 countries. CA Cancer J Clin 68: 394-424, 2018.

2. Cisło M, Filip AA, Arnold Offerhaus GJ, Ciseł B, Rawicz-Pruszyński K, Skierucha M and Polkowski WP: Distinct molecular subtypes of gastric cancer: From Laurén to molecular pathology. Oncotarget 9: 19427-19442, 2018.

3. Ma J, Shen H, Kapesa L and Zeng S: Lauren classification and individualized chemotherapy in gastric cancer. Oncol Lett 11: 2959-2964, 2016.

4. Dittmar Y and Settmacher U: Individualized treatment of gastric cancer: Impact of molecular biology and pathohistological features. World J Gastrointest Oncol 7: 292-302, 2015.

5. Quéro L, Guillerm S and Hennequin C: Neoadjuvant or adjuvant therapy for gastric cancer. World J Gastrointest Oncol 7: 102-110, 2015.

6. Roukos DH and Kappas AM: Perspectives in the treatment of gastric cancer. Nat Clin Pract Oncol 2: 98-107, 2005.

7. Shirabe K, Shimada M, Matsumata T, Higashi H, Yakeishi Y, Wakiyama S, Ikeda Y, Ezaki T, Fukuzawa S, Takenaka K, et al: Analysis of the prognostic factors for liver metastasis of gastric cancer after hepatic resection: A multi-institutional study of the indications for resection. Hepatogastroenterology 50: 1560-1563, 2003.

8. Kanat $\mathrm{O}$ and $\mathrm{O}$ 'Neil BH: Metastatic gastric cancer treatment: A little slow but worthy progress. Med Oncol 30: 464, 2013.

9. Wagner AD, Unverzagt S, Grothe W, Kleber G, Grothey A Haerting J and Fleig WE: Chemotherapy for advanced gastric cancer. Cochrane Database Syst Rev: CD004064, 2010.

10. Wagner AD, Syn NL, Moehler M, Grothe W, Yong WP, Tai BC, Ho $J$ and Unverzagt S: Chemotherapy for advanced gastric cancer. Cochrane Database Syst Rev 8: CD004064, 2017.

11. Apicella M, Corso S and Giordano S: Targeted therapies for gastric cancer: Failures and hopes from clinical trials. Oncotarget 8: 57654-57669, 2017.

12. Lee JS, Kim SH, Im SA, Kim MA and Han JK: Human epidermal growth factor receptor 2 expression in unresectable gastric cancers: Relationship with CT characteristics. Korean J Radiol 18: 809-820, 2017.

13. Liu Y, Ling Y, Qi Q, Zhu M, Wan M, Zhang Y and Zhang C: Trastuzumab increases the sensitivity of HER2-amplified human gastric cancer cells to oxaliplatin and cisplatin by affecting the expression of telomere-associated proteins. Oncol Lett 9: 999-1005, 2015.

14. Pazo Cid RA and Antón A: Advanced HER2-positive gastric cancer: Current and future target therapies. Curr Rev Oncol Hematol 85: 350-362, 2013.

15. Rodrigues dos Santos C, Domingues G, Matias I, Matos J, Fonseca I, de Almeida JM and Dias S: LDL-cholesterol signaling induces breast cancer proliferation and invasion. Lipids Health Dis 13: 16, 2014.

16. Huang B, Song B and Xu C: Cholesterol metabolism in cancer: Mechanisms and therapeutic opportunities. Nat Metab 2: 132-141, 2020.

17. Göbel A, Rauner M, Hofbauer LC and Rachner TD: Cholesterol and beyond-the role of the mevalonate pathway in cancer biology. Biochim Biophys Acta Rev Cancer 1873: 188351, 2020.

18. Wang R, Bi J, Ampah KK, Ba X, Liu W and Zeng X: Lipid rafts control human melanoma cell migration by regulating focal adhesion disassembly. Biochim Biophys Acta 1833: 3195-3205, 2013. 
19. Bathaie SZ, Ashrafi M, Azizian M and Tamanoi F: Mevalonate pathway and human cancers. Curr Mol Pharmacol 10: 77-85, 2017.

20. Clendening JW and Penn LZ: Targeting tumor cell metabolism with statins. Oncogene 31: 4967-4978, 2012.

21. Sharpe LJ and Brown AJ: Controlling cholesterol synthesis beyond 3-hydroxy-3-methylglutaryl-CoA reductase (HMGCR). J Biol Chem 288: 18707-18715, 2013.

22. Iannelli F, Lombardi R, Milone MR, Pucci B, De Rienzo S, Budillon A and Bruzzese F: Targeting mevalonate pathway in cancer treatment: Repurposing of statins. Recent Pat Anticancer Drug Discov 13: 184-200, 2018.

23. Ahmadi Y, Ghorbanihaghjo A and Argani H: The balance between induction and inhibition of mevalonate pathway regulates cancer suppression by statins: A review of molecular mechanisms. Chem Biol Interact 273: 273-285, 2017.

24. Yoshioka H, Coates JW, Chua NK, Hashimoto Y, Brown AJ and Ohgane K: A key mammalian cholesterol synthesis enzyme, squalene monooxygenase, is allosterically stabilized by its substrate. Proc Natl Acad Sci USA 117: 7150-7158, 2020.

25. Gill S, Stevenson J, Kristiana I and Brown AJ: Cholesterol-dependent degradation of squalene monooxygenase, a control point in cholesterol synthesis beyond HMG-CoA reductase. Cell Metab 13: 260-273, 2011.

26. Bernstein DL, Le Lay JE, Ruano EG and Kaestner KH: TALE-mediated epigenetic suppression of CDKN2A increases replication in human fibroblasts. JClin Invest 125: 1998-2006, 2015.

27. Dong W, Vuletic S and Albers JJ: Differential effects of simvastatin and pravastatin on expression of Alzheimer's disease-related genes in human astrocytes and neuronal cells. J Lipid Res 50: 2095-2102, 2009.

28. Shen YY, Yuan Y, Du YY and Pan YY: Molecular mechanism underlying the anticancer effect of simvastatin on MDA-MB-231 human breast cancer cells. Mol Med Rep 12: 623-630, 2015.

29. Buranrat B, Suwannaloet W and Naowaboot J: Simvastatin potentiates doxorubicin activity against MCF-7 breast cancer cells. Oncol Lett 14: 6243-6250, 2017.

30. Lee WS, Chen RJ, Wang YJ, Tseng H, Jeng JH, Lin SY, Liang YC, Chen $\mathrm{CH}$, Lin $\mathrm{CH}$, Lin JK, et al: In vitro and in vivo studies of the anticancer action of terbinafine in human cancer cell lines: G0/G1p53-associated cell cycle arrest. Int J Cancer 106: 125-137, 2003.

31. Ho PY,Zhong WB, Ho YS and Lee WS: Terbinafine inhibits endothelial cell migration through suppression of the Rho-mediated pathway. Mol Cancer Ther 5: 3130-3138, 2006.

32. Vichai V and Kirtikara K: Sulforhodamine B colorimetric assay for cytotoxicity screening. Nat Protoc 1: 1112-1116, 2006.

33. Riihimäki M, Thomsen H, Hemminki A, Sundquist $K$ and Hemminki K: Comparison of survival of patients with metastases from known versus unknown primaries: Survival in metastatic cancer. BMC Cancer 13: 36, 2013.

34. Ge S, Xia X, Ding C, Zhen B, Zhou Q, Feng J, Yuan J, Chen R, $\mathrm{Li} \mathrm{Y}, \mathrm{Ge} \mathrm{Z}$, et al: A proteomic landscape of diffuse-type gastric cancer. Nat Commun 9: 1012, 2018.

35. Gunturu KS, Woo Y, Beaubier N, Remotti HE and Saif MW: Gastric cancer and trastuzumab: First biologic therapy in gastric cancer. Ther Adv Med Oncol 5: 143-151, 2013.

36. Kim SM and Park SH: Chemotherapy beyond second-line in advanced gastric cancer. World J Gastroenterol 21: 8811-8816, 2015.

37. Shah MA and Schwartz GK: Cell cycle-mediated drug resistance: An emerging concept in cancer therapy. Clin Cancer Res 7: 2168-2181, 2001
38. Basque JR, Chénard M, Chailler P and Ménard D: Gastric cancer cell lines as models to study human digestive functions. J Cell Biochem 81: 241-251, 2001.

39. Clendening JW, Pandyra A, Li Z, Boutros PC, Martirosyan A, Lehner R, Jurisica I, Trudel S and Penn LZ: Exploiting the mevalonate pathway to distinguish statin-sensitive multiple myeloma. Blood 115: 4787-4797, 2010.

40. Caruso MG, Notarnicola M, Cavallini A and Di Leo A 3-Hydroxy-3-methylglutaryl coenzyme A reductase activity and low-density lipoprotein receptor expression in diffuse-type and intestinal-type human gastric cancer. J Gastroenterol 37: 504-508, 2002.

41. Kim ST, Kang JH, Lee J, Park HP, Park O, Park YS, Lim Y, Hwang G, Lee SC, Park KW, et al: Simvastatin plus capecitabine-cisplatin versus placebo plus capecitabine-cisplatin in patients with previously untreated advanced gastric cancer: A double-blind randomized phase 3 study. Eur J Cancer 50: 2822-2830, 2014

42. Lytras T, Nikolopoulos G and Bonovas S: Statins and the risk of colorectal cancer: An updated systematic review and meta-analysis of 40 studies. World J Gastroenterol 20: 1858-1870, 2014.

43. Jones P, Kafonek S, Laurora I and Hunninghake D: Comparative dose efficacy study of atorvastatin versus simvastatin, pravastatin, lovastatin, and fluvastatin in patients with hypercholesterolemia (The CURVES Study). Am J Cardiol 81: 582-587, 1998.

44. Gerson RJ, MacDonald JS, Alberts AW, Kornbrust DJ, Majka JA, Subbs R and Bokelman DL: Animal safety and toxicology of simvastatin and related hydroxy-methyl glutaryl-Coenzyme A reductase inhibitors. Am J Med 87: 28S-38S, 1989.

45. Wong WW, Clendening JW, Martirosyan A, Boutros PC, Bros C, Khosravi F, Jurisica I, Stewart AK, Bergsagel PL and Penn LZ: Determinants of sensitivity to lovastatin-induced apoptosis in multiple myeloma. Mol Cancer Ther 6: 1886-1897, 2007.

46. Liao JK and Laufs U: Pleiotropic effects of statins. Annu Rev Pharmacol Toxicol 45: 89-118, 2005.

47. Mahoney CE, Pirman D, Chubukov V, Sleger T, Hayes S, Fan ZP, Allen EL, Chen Y, Huang L, Liu M, et al: A chemical biology screen identifies a vulnerability of neuroendocrine cancer cells to SQLE inhibition. Nat Commun 10: 96, 2019.

48. Al-Haidari AA, Syk I and Thorlacius H: HMG-CoA reductase regulates CCL17-induced colon cancer cell migration via geranylgeranylation and RhoA activation. Biochem Biophys Res Commun 446: 68-72, 2014

49. Chushi L, Wei W, Kangkang X, Yongzeng F, Ning $X$ and Xiaolei C: HMGCR is up-regulated in gastric cancer and promotes the growth and migration of the cancer cells. Gene 587: 42-47, 2016.

50. Ebert K, Mattes J, Kunzke T, Zwingenberger G and Luber B: MET as a resistance factor for afatinib therapy and motility driver in gastric cancer cells. PLoS One 14: e0223225, 2019.

51. Sethunath V, Hu H, De Angelis C, Veeraraghavan J, Qin L, Wang N, Simon LM, Wang T, Fu X, Nardone A, et al: Targeting the mevalonate pathway to overcome acquired anti-HER2 treatment resistance in breast cancer. Mol Cancer Res 17: 2318-2330, 2019.

c) (i) $\Theta$ This work is licensed under a Creative Commons Attribution-NonCommercial-NoDerivatives 4.0 International (CC BY-NC-ND 4.0) License. 